\title{
The leukodystrophy mutation Polr3b R103H causes homozygote mouse embryonic lethality and impairs RNA polymerase III biogenesis
}

\author{
Karine Choquet ${ }^{1,2,3}$, Maxime Pinard ${ }^{4}$, Sharon Yang ${ }^{1}$, Robyn D. Moir ${ }^{5}$, Christian Poitras ${ }^{4}$, Marie-Josée Dicaire ${ }^{1}$, \\ Nicolas Sgarioto ${ }^{1}$, Roxanne Larivière ${ }^{1}$, Claudia L. Kleinman ${ }^{2,3}$, Ian M. Willis ${ }^{5}$, Marie-Soleil Gauthier ${ }^{4}$, \\ Benoit Coulombe $e^{4,6}$ and Bernard Brais ${ }^{1,2^{*}}$ (D)
}

\begin{abstract}
Recessive mutations in the ubiquitously expressed POLR3A and POLR3B genes are the most common cause of POLR3-related hypomyelinating leukodystrophy (POLR3-HLD), a rare childhood-onset disorder characterized by deficient cerebral myelin formation and cerebellar atrophy. POLR3A and POLR3B encode the two catalytic subunits of RNA Polymerase III (Pol III), which synthesizes numerous small non-coding RNAs. We recently reported that mice homozygous for the Polr3a mutation c.2015G > A (p.Gly672Glu) have no neurological abnormalities and thus do not recapitulate the human POLR3-HLD phenotype. To determine if other POLR3-HLD mutations can cause a leukodystrophy phenotype in mouse, we characterized mice carrying the Polr3b mutation c.308G > A (p.Arg103His). Surprisingly, homozygosity for this mutation was embryonically lethal with only wild-type and heterozygous animals detected at embryonic day 9.5. Using proteomics in a human cell line, we found that the POLR3B R103H

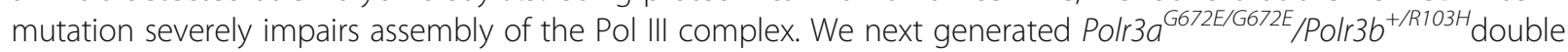
mutant mice but observed that this additional mutation was insufficient to elicit a neurological or transcriptional phenotype. Taken together with our previous study on Polr3a G672E mice, our results indicate that missense mutations in Polr3a and Polr3b can variably impair mouse development and Pol III function. Developing a proper model of POLR3-HLD is crucial to gain insights into the pathophysiological mechanisms involved in this devastating neurodegenerative disease.
\end{abstract}

Keywords: Leukodystrophy, RNA polymerase III, Mouse model, POLR3A, POLR3B, Myelination

\section{Background}

Recessive mutations in POLR3A, POLR3B, POLR1C and POLR3K cause RNA Polymerase III-related hypomyelinating leukodystrophy (POLR3-HLD), [1-4] a devastating childhood-onset neurodegenerative disorder characterized by motor regression, cerebellar features and/or cognitive dysfunction, as well as hypomyelination and cerebellar atrophy on magnetic resonance imaging (MRI) [5]. To date,

\footnotetext{
* Correspondence: bernard.brais@mcgill.ca

'Montreal Neurological Institute, McGill University, 3801 University Street, room 622, Montréal, Québec H3A 2B4, Canada

²Department of Human Genetics, McGill University, Québec, Montréal, Canada

Full list of author information is available at the end of the article
}

more than 100 POLR3-HLD cases have been reported. The majority of these involve mutations that are present in a single family or in only a handful of patients [5]. There is significant phenotypic heterogeneity regarding severity, age of onset and nature of symptoms and no clear genotype-phenotype correlation has been observed [5]. POLR3A and POLR3B encode the two catalytic subunits of RNA Polymerase III (Pol III), an essential enzyme responsible for the synthesis of nuclear-encoded transfer RNAs (tRNA) and various other small housekeeping noncoding RNAs (ncRNA). However, none of these Pol III transcripts have a known role in myelination and/or cerebellar function, highlighting the need for a disease model in which the pathophysiological mechanisms responsible

(c) The Author(s). 2019 Open Access This article is distributed under the terms of the Creative Commons Attribution 4.0 International License (http://creativecommons.org/licenses/by/4.0/), which permits unrestricted use, distribution, and 
for POLR3-HLD can be investigated. We recently reported that mice homozygous for the French Canadian founder mutation Polr3a c.2015G > A (p.Gly672Glu) have normal motor function, myelination and cerebellar integrity and thus do not recapitulate the human POLR3-HLD phenotype [6]. Nonetheless, it remains unknown whether other mutations in Polr3a or Polr3b can lead to a POLR3HLD phenotype in mice.

In this follow-up study, we assessed the impact of a different POLR3-HLD-causing mutation, Polr3b c.308G > A (p.Arg103His), and found that it has a severe impact on mouse development and on Pol III biogenesis, indicating that mutations in Pol III subunits have variable effects in mice that may be a consequence of their specific defects on Pol III function. Second, we show that increasing the Pol III mutational burden of Polr3a G672E mice by adding a heterozygous Polr $3 b$ mutation does not lead to significant impairment at the phenotypic, histological or transcriptional levels, indicating that one functional Polr3b allele is sufficient to maintain normal Pol III function even in the presence of two mutated Polr3a alleles, providing further evidence that the Polr3a G672E mutation mimics a wild-type allele in mouse and that vulnerability to Pol III mutations varies between species.

\section{Results and discussion}

To determine if other mutations in Polr $3 a$ or Polr $3 b$ cause a leukodystrophy phenotype in mice, we acquired heterozygous Polr3b c.308G > A (p.Arg103His) mice, which were generated using CRISPR-Cas9. Since this knock-in mouse was being produced in parallel with Polr $3 b^{-/-}$mice as part of the NorCOMM2 project, the c.308G > A mutation was selected because of its proximity to the targeting site of the single guide RNA used to generate $\mathrm{Polr}_{3 b^{--}}$mice. This mutation has only been observed in one POLR3-HLD patient in compound heterozygosity with POLR3B c.1568C > T (p.Val523Glu) [5]. We confirmed the presence of the heterozygous c.308G > A mutation in these animals by Sanger sequencing of tail genomic DNA (Fig. 1a). However, when we bred heterozygous mice together, we were unable to obtain homozygous Polr $3 b^{R 103 H / R 103 H}$ mice. After weaning,

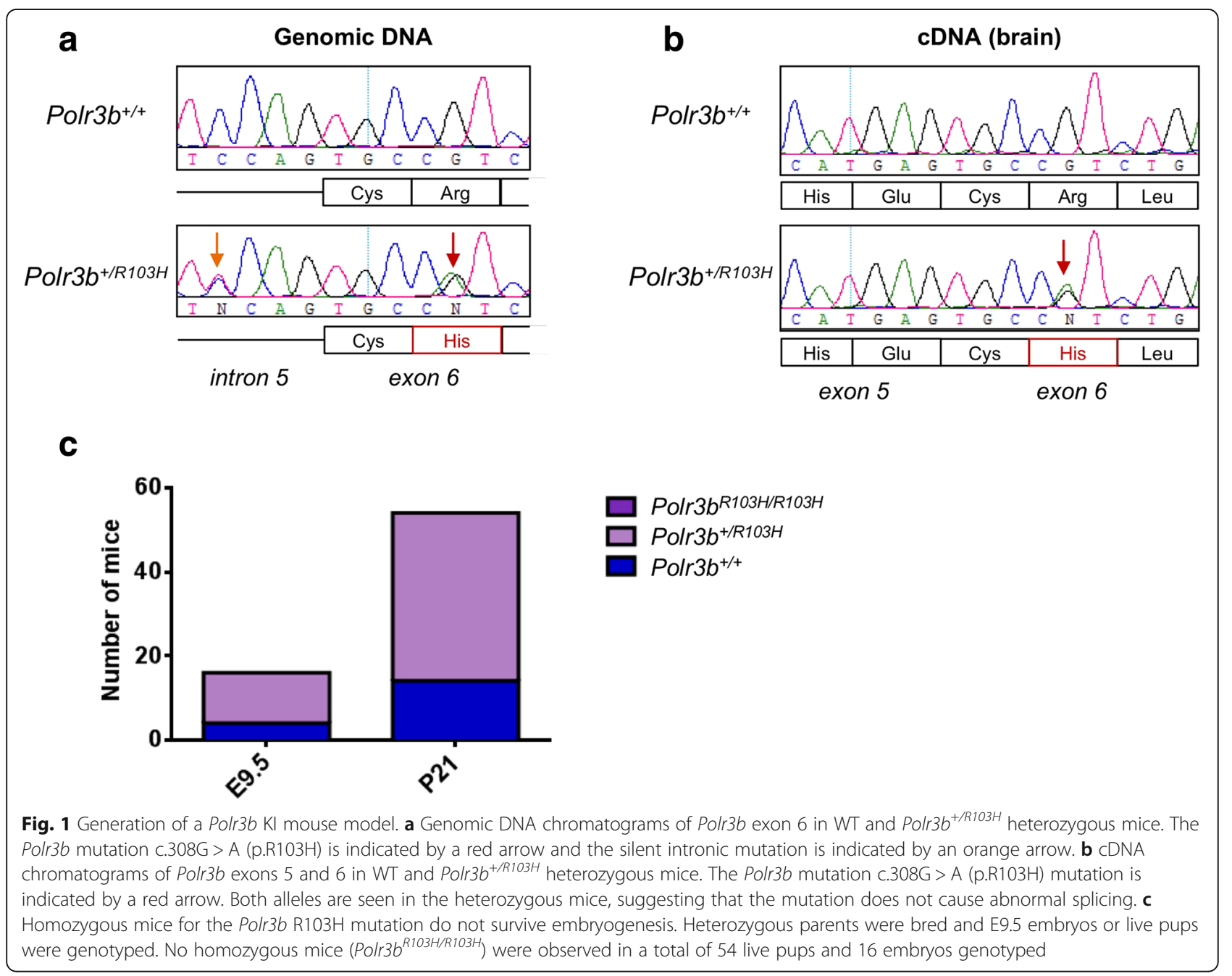


only wild-type (WT) (14/54) and heterozygote animals (40/54) were detected (Fig. 1c). A similar distribution of genotypes was seen at embryonic day 9.5 (Fig. 1c). These results indicate that the Polr3b R103H mutation is embryonically lethal at or before mid-gestation, similarly to Polr $3 a^{-1-}$ null mice [6]. This is in stark contrast to homozygous Polr3a ${ }^{\text {G672E/G672E }}$ mice, which are viable and do not display any abnormalities at 12 months of age [6]. POLR3A and POLR3B protein sequences are highly conserved between human and mouse, with perfect conservation surrounding the POLR3A G672 and POLR3B R103 sites (multiple protein alignment by Clustal Omega) $[6,7]$. Thus, the phenotypic differences between the two mouse models cannot be explained by differences in sequence conservation levels between species.

The c.308G > A mutation is located near the start of exon 6 and was introduced alongside an intronic mutation (c.304-4C > T) to prevent CRISPR-Cas9 re-editing of the repaired genomic DNA. To verify whether these mutations cause aberrant splicing leading to a null allele, we performed RT-PCR and Sanger sequencing of exons 4-8 of the Polr3b cDNA in heterozygous mice. Normal splicing was confirmed (Fig. 1b). Furthermore, both alleles were detected in similar proportions on the chromatogram, indicating that the mutant allele is not degraded. Thus, the c.308G > A mutation does not have a detrimental impact on mRNA splicing or stability. Taken together, results from Polr3a $a^{\mathrm{G} 672 \mathrm{E} / \mathrm{G} 672 \mathrm{E}}$ and Polr $3 b^{+/ R 103 H}$ mice may suggest that human missense mutations in Polr3a and Polr3b can have no impact in mice or lead to early developmental arrest.

We previously demonstrated that the POLR3A G672E mutation has a mild impact on Pol III function in human cells, since it does not impair Pol III complex assembly or nuclear import and only leads to a mild decrease in POLR3A occupancy at target loci [6]. Considering the severity of the Polr $3 b \mathrm{R} 103 \mathrm{H}$ mutation in mice, we hypothesized that this mutation would have a more detrimental effect on Pol III function. To test this hypothesis, FLAG-tagged versions of the wild-type (WT) or mutant $(\mathrm{R} 103 \mathrm{H})$ forms of POLR3B were transiently expressed in HEK293 cells. Whole cell extracts were affinity purified using an anti-FLAG antibody and analyzed by label-free shotgun proteomics (AP-MS). [3] FLAG-tagged WT and R103H POLR3B expression levels (Additional File 1: Figure S1A-B) and pulled-down levels (Additional File 2: Table S1) were similar and POLR3B interactors (prey proteins) levels were normalized by the pulled-down levels of the bait (POLR3B) in each purification. We observed that the POLR3B R103H mutation greatly impedes the assembly or stability of the Pol III complex. Indeed, eleven of the sixteen remaining Pol III subunits were detected in pulldowns that used tagged WT POLR3B whereas lower amounts of each of these subunits relative to WT were pulled down using POLR3B R103H (Fig. 2a, Additional File 2: Table S1). The ratio of six of these subunits showed a statistically significant reduction in the mutant purifications compared to that of the WT (Fig. 2b), implying a lower affinity of the mutant POLR3B subunit for its partners in vivo and a decreased abundance of the mutant Pol III complex. These data suggest that in homozygous mouse cells, the defective Pol III biogenesis conferred by the mutant POLR3B subunit may underlie the impaired mouse embryonic development phenotype. Importantly, when combined with our previous study [6], our data suggest that mutations causing a milder effect on Pol III function, such as POLR3A G672E, may not lead to a phenotype in mice, perhaps because of inter-species differences in myelination [6]. On the other hand, some mutations that affect Pol III complex assembly or nuclear import and that are generally seen as compound heterozygous alleles in humans may be too severe in the homozygous state, thus preventing embryonic development. Indeed, numerous POLR3-HLD cases with compound heterozygous alleles are known to include one allele with a frameshift or nonsense mutation [5]. Since we have only studied two mutations thus far, further experiments on other POLR3A and POLR3B mutations will be necessary to confirm such a correlation. This does not preclude that other mutations, perhaps ones that have a moderate effect on chromatin occupancy or transcription elongation, might lead to a clinical mouse phenotype recapitulating the human disease. We recently showed that in human cell lines, the POLR3HLD-causing POLR3A M862 V mutation does not impair Pol III complex assembly or nuclear import but leads to decreased levels of a subset of Pol III transcripts, including tRNAs and 7SL RNA [8]. Thus, this mutation appears to have an intermediate functional phenotype in human cells that could perhaps lead to a neurological phenotype in mouse. Examining the functional impact of a larger panel of mutations in human cells could help pinpoint those that may produce a mouse phenotype, though mouse brain development may be less susceptible than the human brain to the impact of such mutations.

Considering the severe impact of the Polr $3 b \mathrm{R} 103 \mathrm{H}$ mutation on mouse development, we next asked whether introducing this mutation in the heterozygous state in Polr3a ${ }^{\mathrm{G} 672 E / G 672 E}$ mice, thus increasing the Pol III mutational burden, could lead to a phenotype that would resemble POLR3-HLD. We crossed homozygous Polr $3 a^{\mathrm{G} 672 \mathrm{E} / \mathrm{G} 672 \mathrm{E}}$ mice with heterozygous Polr $3 b^{+/ R 103 \mathrm{H}}$ mice and the resulting pups were bred to obtain double mutant mice (Polr3a $a^{\text {G672E/G672E }} /$ Polr $3 b^{+/ R 103 H}$, hereafter referred to as DM mice) (Fig. 3a). DM mice were viable, reproduced normally and did not display a grossly 


\section{a}

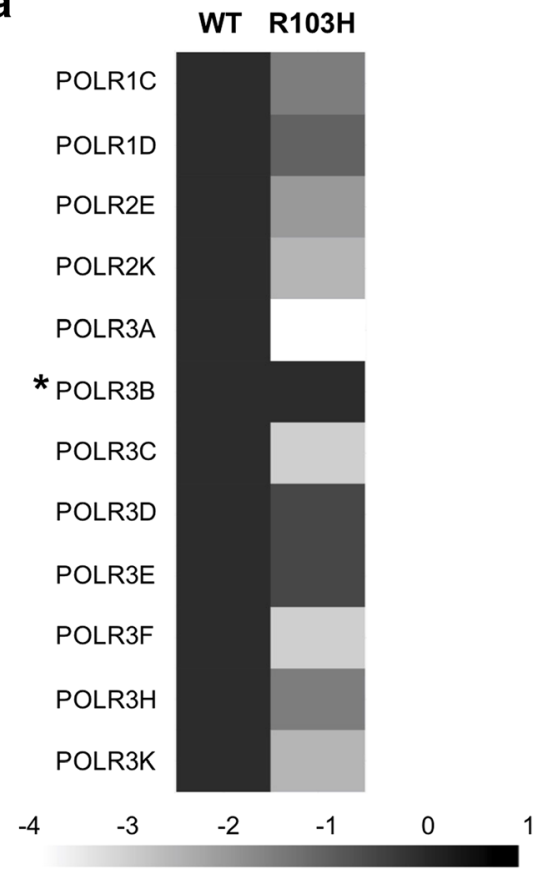

b

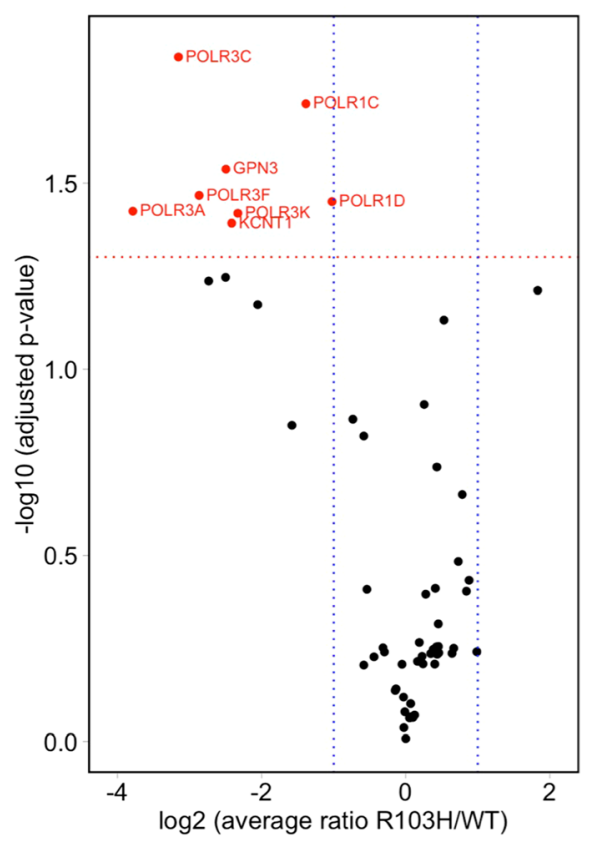

Fig. 2 Impact of POLR3B R103H mutation on the assembly of Pol III complex. FLAG-tagged POLR3B WT or R103H were expressed in HEK293 cells, purified using an anti-FLAG antibody and digested with trypsin. The co-purified proteins were identified by LC-MS/MS mass spectrometry. The

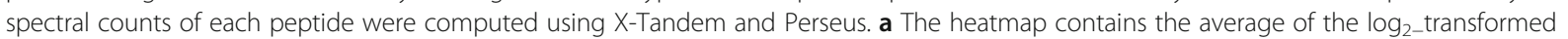
spectral count ratios across all 4 replicates. POLR3B (the bait) is identified by an asterisk. $\mathbf{b}$ Volcano plot of the $\log _{2}$-transformed average spectral count ratio $\mathrm{R} 103 \mathrm{H} / \mathrm{NT}$ ( $\mathrm{x}$-axis) and the - $\log _{10} \mathrm{q}$-value obtained by using a two-tailed T-test adjusted with a permutation-based multiple hypothesis testing and an $\mathrm{s} 0$ correction factor of 0.1 (y-axis). Proteins marked in red are considered statistically significant

abnormal phenotype. To evaluate balance and coordination, we performed the balance beam test at 6 months of age. We did not detect statistically significant differences in latency to cross the beam or in the number of foot slips between WT and DM mice, suggesting that DM mice do not have impaired gait or cerebellar ataxia (Fig. 3b). Furthermore, WT and DM mice performed comparably in the open field test at 6 months of age, indicating normal general locomotion and voluntary movement (Fig. 3c). We next assessed the presence of hypomyelination and cerebellar atrophy, which are the main pathological features of POLR3-HLD. [5, 9] Coronal brain sections from six month old DM mice stained with an antibody against Proteolipid protein (PLP), one of the main protein components of myelin, were indistinguishable from age-matched WT mice, suggesting that DM mice undergo complete myelination (Fig. 4a). Moreover, Nissl staining and Purkinje cell counts showed normal cerebellar integrity in DM mice (Fig. 4b). Altogether, our behavioral and histological data indicate that DM mice do not recapitulate the phenotypic or pathological features of POLR3-HLD at 6 months of age, and thus that the heterozygous Polr $3 b$ c.308G > A mutation does not lead to a phenotype in Pol$r 3 a^{\mathrm{G} 672 \mathrm{E} / \mathrm{G} 672 \mathrm{E}}$ mice. Lastly, we evaluated the transcriptional impact of Polr3a and Polr3b mutations in the brain of DM mice. Using RNA-sequencing (RNA-seq), we found that Polr3a and Polr3b mRNA levels were comparable in WT and DM mice (Fig. 5a), providing further evidence that the two missense mutations do not alter mRNA stability. Furthermore, the three Pol III transcripts detected by this technique (Rpph1, Rmrp and Rn7sk) appeared unaffected by the Polr3a and Polr $3 b$ mutations (Fig. $5 \mathrm{~b}$ ). In addition, no protein-coding genes showed significant differences (adjusted $p$-value $<0.05$ and absolute $\log 2$ fold change $>1$ ) between the two groups, demonstrating that Polr3a G672E and Polr3b R103H missense mutations do not have a global impact on the brain transcriptome. We also measured the levels of one precursor tRNA and one mature tRNA by Northern Blot and found no change in their amounts in WT and DM mice (Fig. 5c), suggesting that $50 \%$ wild-type Polr $3 b$ is sufficient to maintain Pol III function. Thus, our findings strongly suggest that adding a heterozygous mutation in a second Pol III subunit is insufficient to impair mouse neurological function or Pol III transcript levels in Polr3a ${ }^{\mathrm{G} 672 \mathrm{E} / \mathrm{G} 672 \mathrm{E}}$ mice at six months of age.

Characterization of Polr3a ${ }^{\mathrm{G} 672 \mathrm{E} / \mathrm{G} 672 \mathrm{E}}$, Polr $3 b^{\text {R103H/R103H }}$ and DM mice clearly shows that the impact of Pol III mutations differs in human and mice. Whether this is due to inter-species differences in myelination, to variable Pol III 
a

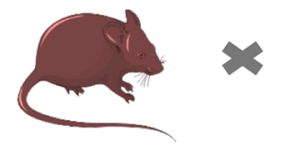

Polr3a $a^{\mathrm{G} 672 \mathrm{E} / \mathrm{G} 672 \mathrm{E}}$

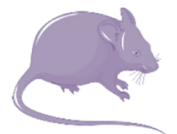

Polr $3 b^{+/ R 103 H}$

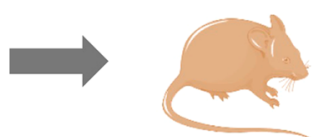

Double mutant (DM) Polr3a $a^{G 672 E / G 672 E} ;$ Polr3b $b^{+/ R 103 H}$ b
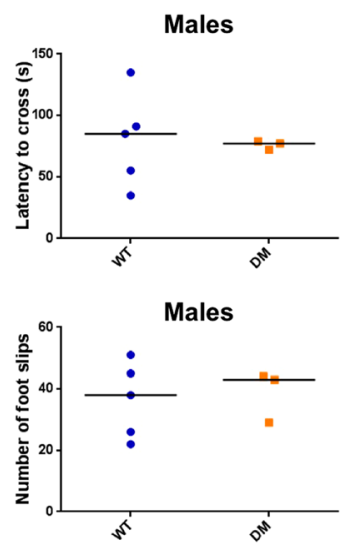

d

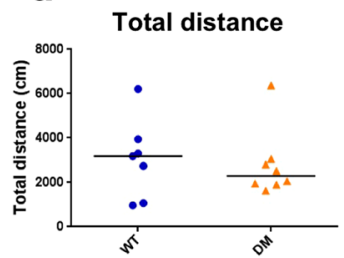

Females

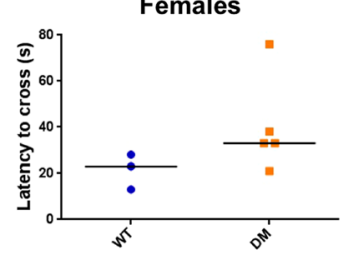

Females

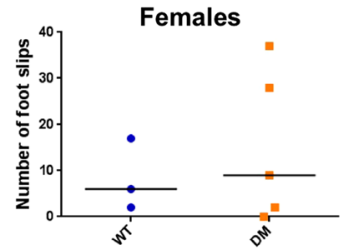

C
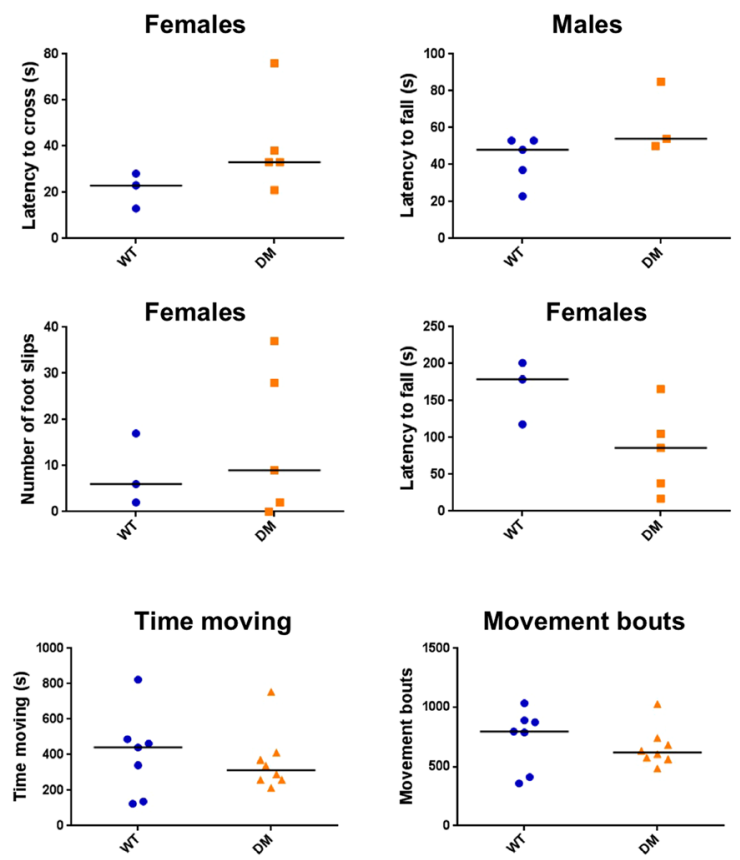

Fig. 3 Normal motor function in double mutant mice. a Polr3a $a^{G 672 E / G 672 E}$ and Polr3b $b^{+/ R 103 H}$ mice were crossed to generate double mutant mice that are homozygous for the Polr3a G672E mutation and heterozygous for the Polr3b R103H mutation. b Results from the 6 mm beam test at 6 months of age in males and females. Latencies to cross (top) and number of foot slips (bottom) are shown. For each mouse, three trials were performed and summed. $\mathbf{c}$ Results from the rotarod at 6 months of age in males and females. For each mouse, three trials were performed and summed. $\mathbf{d}$ Results from the open field test at 6 months of age in an independent cohort of males only. The open field test was run for 90 min per mouse during which total distance traveled (left), total time spent moving (middle) and number of movement bouts (right) were recorded for each 10 min interval

function or regulation, or to the existence of speciesspecific Pol III transcripts remains unclear. As evidence for the latter, the most affected Pol III transcript detected in several POLR3-HLD human cellular models, including patient-derived fibroblasts, is BC200 RNA [8], suggesting a role for this primate-specific RNA in POLR3-HLD pathophysiology [10]. BC200 RNA has a functional analog in mouse, Bc1 RNA, which is also synthesized by Pol III. While both transcripts play a similar role in translational regulation, they have different evolutionary origins [10] and recent evidence indicates that BC200 RNA is also involved in mRNA splicing and stability $[11,12]$. We found that BC200 RNA was downregulated in patient fibroblasts harboring the POLR3A G672E mutation [8], while Bc1 RNA was not affected in mouse brains homozygous for the same mutation [6], further suggesting that the two transcripts may have different sensitivities to Pol III hypofunction that could explain the absence of neurological phenotype in mice. $B c 1$ knockout (KO) in mouse does not lead to hypomyelination or neurological deficits [13]. In contrast, in a human oligodendroglial cell line, both the POLR3A M852 V mutation and BC200 RNA KO led to decreased Myelin basic protein (MBP) mRNA levels compared to wild-type cells upon cellular differentiation, indicating that Pol III hypofunction could have a detrimental impact on myelin biogenesis and that this effect may be exerted through impaired BC200 RNA expression [8]. Nonetheless, the effect on $M B P$ expression was milder in BC200 KO compared to POLR3A-mutated cells, suggesting that multiple factors are at play in the latter and that other Pol III transcripts such as tRNAs, which are conserved in mouse, could contribute to POLR3-HLD.

While it is possible that POLR3-HLD simply cannot be properly modeled in mice, other genetic, developmental or environmental factors that are absent in laboratory mice may be at play in human cases and contribute to pathogenesis. For instance, in addition to its role in nuclear gene transcription, Pol III also acts as 
a
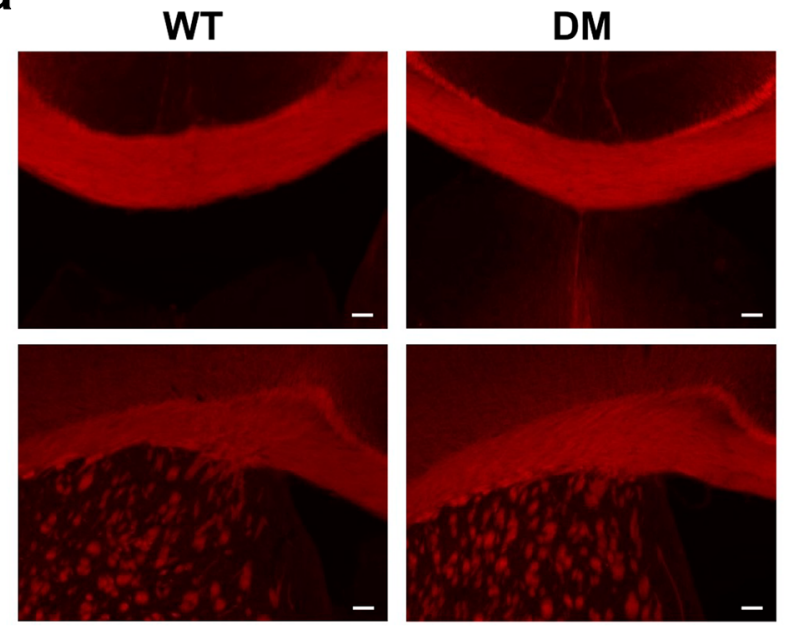

b
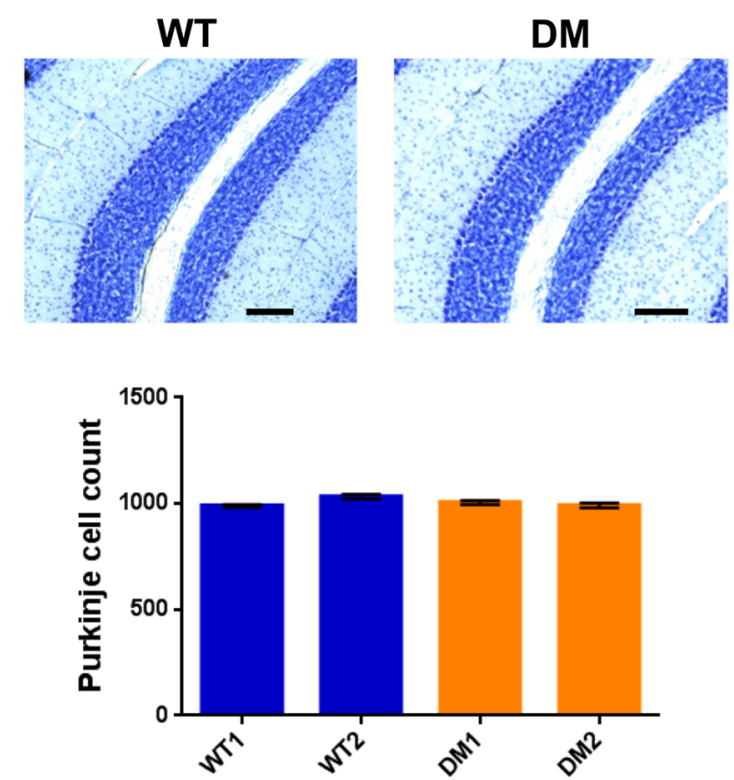

Fig. 4 Normal myelination and cerebellar integrity in double mutant mice at 6 months of age. a Immunofluorescence for proteolipid protein (PLP) showing normal staining in the corpus callosum (top: coronal view; bottom: sagittal view) of DM mice compared to WT. Staining was performed on three mice per group and representative images are shown for each group. Scale bar: $100 \mu \mathrm{m}$. b Top: Nissl staining of sagittal cerebellar sections performed on two mice per group. Representative images are shown for each group. Scale bar: 100 um. Bottom: Purkinje cell counts of mid-sagittal cerebellar sections. Data are represented as mean $+/-$ SEM of three sections per mouse

a DNA sensor in innate immunity [14]. Since approximately half of POLR3-HLD patients undergo neurological deterioration upon infections, [5] exposure to certain pathogens may play a role in disease progression via this lesser-known function of Pol III.

In conclusion, this study further highlights the difficulty of establishing an appropriate mouse model for POLR3-HLD. Nonetheless, the fact that the Polr $3 b^{\mathrm{R} 103 \mathrm{H} / \mathrm{R} 103 \mathrm{H}}$ mice are embryonic lethal while the Polr3a ${ }^{\text {G672E/G672E }}$ mice have no CNS phenotype raises the possibility that other mutations in genes encoding Pol III subunits could lead to intermediate phenotypes that would resemble POLR3-HLD. Furthermore, the possible correlation between mouse phenotype and impact on Pol III function provides a useful tool to select such candidate mutations.

\section{Materials and methods}

\section{Animals}

All experiments were carried out according to good practice of handling laboratory animals consistent with the Canadian Council on Animal Care and approved by the University Animal Care Committee. The mouse line C57BL/6 N-Polr3b $<$ em4Tcp $>\left(\right.$ Polr $\left.3 b^{+/ R 103 H}\right)$ was made as part of the NorCOMM2 project funded by the Care4Rare Canada Consortium, Genome Canada and the Ontario Genomics Institute (OGI-051) at the Toronto
Centre for Phenogenomics. It was obtained from the Canadian Mouse Mutant Repository. Briefly, the mutant allele was created using CRISPR-Cas9, by injecting the Cas9 protein, single guide RNA ( 5 '-TCATGTCCCTCAGACGGCAC-3') and single-strand oligonucleotide repair template (5' -gtgcactgtattcacagtgggattgctggagggcaggggtcgctcgctcactgtgattctgcttcagTGCCATCTGAGGGACATGACGTACTCC GCCCCAATCACAGTGGACATTGAGTATACCCGAG GCAGCCAGAGGA-3') including the c.308G > A mutation and the intronic mutation c.304-4C $>\mathrm{T}$ to prevent reediting of the properly mutated genomic DNA. For determination of the lethality of Polr $3 b^{R 103 H / R 103 H}$ mice, heterozygous Polr $3 b^{+/ R 103 H}$ mice were interbred and gestating females were sacrificed at E9.5 to extract embryos for genotyping. The generation of Polr3a ${ }^{G 672 E / G 672 E}$ mice was previously described [6]. For the production of DM mice, Polr3a $a^{\text {G672E/G672E }}$ and Polr $3 b^{+/ R 103 H}$ were bred, resulting in $50 \%$ Polr $3 a^{+/ G 672 E} /$ Polr $3 b^{+/ R 103 H}$ and 50\% Polr3a $a^{+/ G 672 E} /$ Polr $3 b^{+/+}$mice. Polr3a $a^{+/ G 672 E} /$ Polr $3 b^{+/ R 103 H}$ males and females were subsequently bred together to generate Polr $3 a$ G672E/G672E $/$ Polr $3 b^{+/ R 103 H}$ DM mice.

\section{Genotyping and sanger sequencing}

Genomic DNA was extracted from tail biopsies or whole embryos using the Gentra Puragene Tissue Kit (Qiagen). Genotyping of the Polr3b R103H allele was performed with two alternative techniques. We first used real-time 

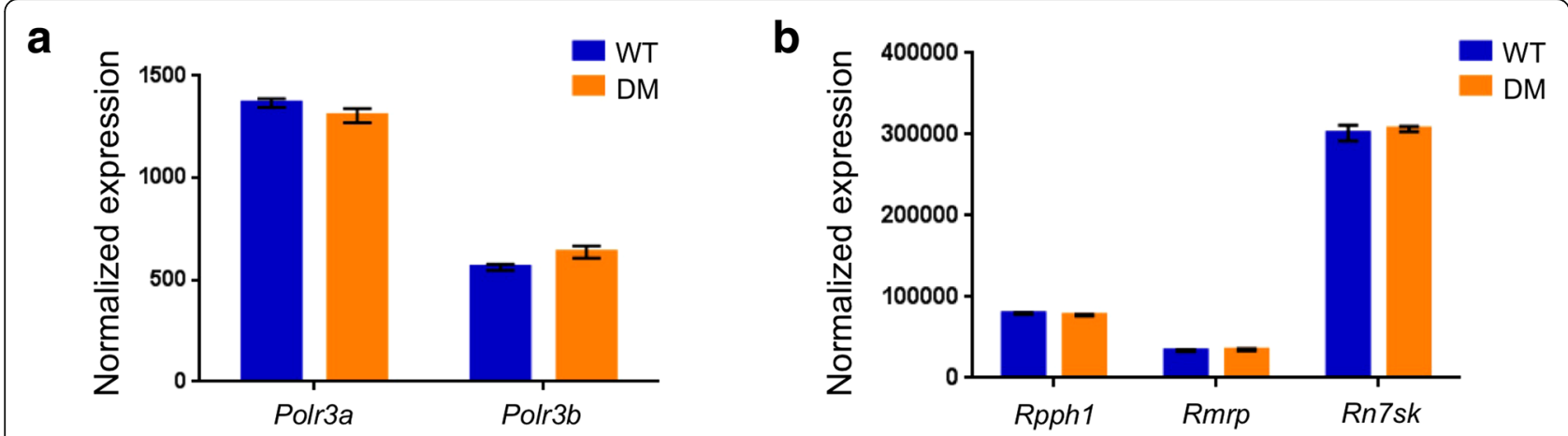

C CEREBRUM CEREBELLUM
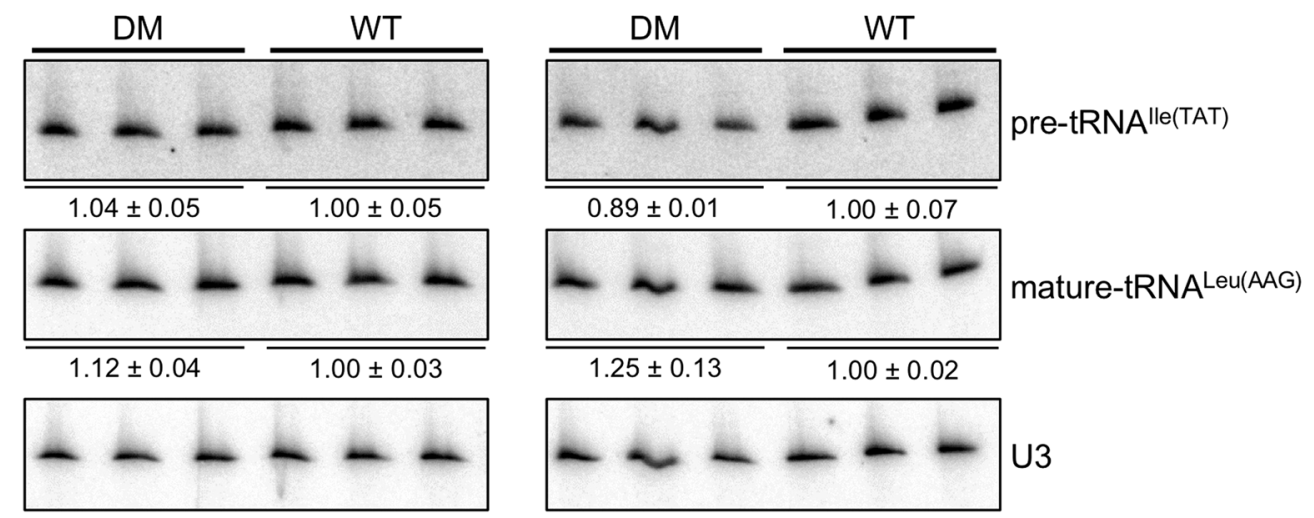

Fig. 5 Normal expression of Polr3a and Polr3b mRNAs and Pol III transcripts in double mutant mice at 6 months of age. a, b Expression levels of a) Polr3a and Polr3b and $\mathbf{b}$ ) three Pol III transcripts measured by RNA-seq in the cerebrum of three WT and three DM mice. Expression was normalized with DESeq2. Data are represented as mean +/- SEM. c Northern blots of precursor (pre) and mature tRNA species from the cerebrum (left) and cerebellum (right) of three WT and three DM mice. U3 snRNA, a Pol II transcript, was used as a loading control. Quantification of mean +/- SEM after normalization are indicated below the blots for each group

PCR allelic discrimination and LNA probes (Integrated DNA Technologies) (forward primer: 5'-GCTC GCTCACTGTGATTCT-3', reverse primer: $5^{\prime}$-CTC GGGTATACTCAATGTCCAC-3', Polr3b WT LNA probe: $\mathrm{HEX}-\mathrm{CT}+\mathrm{CAG}+\mathrm{A}+\mathrm{C}+\mathrm{GGC}+\mathrm{AC}$-IowaBlack FQ, Polr3b R103H LNA probe: FAM-AG + A + T + GGCA+CTGG-IowaBlack FQ). We later used PCR amplification of Polr3b exon 6 (primers: 5'-GCCAAGCACAC ACATGTTCTA-3' and 5'-TACCATTTCCCACACCC TTC-3') followed by digestion with the restriction enzyme BsrI. The presence of the c.308G > A mutation abolishes the BsrI restriction site. Genotyping of the Polr3a G672E allele was conducted as previously reported [6]. For Sanger sequencing of Polr3b exon 6, genomic DNA was amplified by PCR using the following primers: $5^{\prime}$-ATTC ACAGTGGGATTGCTGG-3' and 5' -TGGCTTAAGGTCACATCATGG-3'. PCR products were sequenced at the McGill University and Genome Quebec Innovation Center, using a 3730XL DNA Analyzer (Applied Biosystems). Sequence chromatograms were analyzed using SeqMan v.4.03 (DNASTAR Inc., Madison, WI, USA).

\section{Plasmid construction}

POLR3B WT DNA sequence was amplified by PCR using the following primers (Forward 5'-AAGG AAAAAAGCGGCCGCATGGACGTGCTAGCGGAGG AGTT-3' and Reverse 5' -CTAGTCTAGATTCATTGT ACTTGGACAGTTTTAACC-3') and transferred in a p3XFLAG-CMV-14 expressing vector (Sigma). The c.308G > A (p.R103H) POLR3B mutant was generated by site-directed mutagenesis by amplifying the WTexpressing plasmid with overlapping primers encoding the desired mutation with a Q5 hot-start high-fidelity DNA polymerase (New England Biolabs) and overnight digestion of the parental plasmid with DpnI (New England Biolabs) prior to transformation. All POLR3B plasmid sequences were verified by sequencing. 


\section{Affinity purification coupled with mass spectrometry (AP-MS)}

Human embryonic kidney cell line 293 (HEK293) were maintained in culture in DMEM media supplemented with $10 \%$ fetal bovine serum and $2 \mathrm{mM}$ glutamine, seeded in 6-wells plates at $3 \times 10^{5}$ cells/well, grown overnight and transiently transfected with 500 ng of FLAGtagged WT or R103H POLR3B expressing plasmids by using Jet Prime transfection reagent (PolyPlus) according to manufacturer's instruction. Transfected cells were incubated at $37^{\circ} \mathrm{C}$ for $24 \mathrm{~h}$, washed with sterile PBS, pelleted at 3500RPM and snap frozen in liquid nitrogen. Affinity purifications were performed using standard procedures in four independent replicate experiments [3]. The speedvac protein extracts were then re-solubilized in $10 \mu \mathrm{L}$ of a $6 \mathrm{M}$ urea buffer, reduced in reduction buffer $(45 \mathrm{mM}$ DTT, $100 \mathrm{mM}$ ammonium bicarbonate) for $30 \mathrm{~min}$ at $37^{\circ} \mathrm{C}$, and alkylated in alkylation buffer $(100 \mathrm{mM}$ iodoacetamide, $100 \mathrm{mM}$ ammonium bicarbonate) for $20 \mathrm{~min}$ at $24{ }^{\circ} \mathrm{C}$ in dark and proteins were digested in $10 \mu \mathrm{L}$ of the trypsin solution $(5 \mathrm{ng} / \mu \mathrm{L}$ of trypsin sequencing grade from Promega, $50 \mathrm{mM}$ ammonium bicarbonate) at $37^{\circ} \mathrm{C}$ for 18 h. The protein digests were acidified with trifluoroacetic acid for desalting and removal of residual detergents by MCX (Waters Oasis MCX 96-well Elution Plate) according to manufacturer's instructions. The resulting peptides were identified with tandem mass spectrometry (LC-MS/ MS) using a HPLC system coupled to an Orbitrap Fusion mass spectrometer (Thermo Scientific) through a Nanospray Flex Ion Source. MS/MS raw data were searched against the Uniprot database (updated on June $12^{\text {th }} 2018$ ) using X-Tandem (version 2013.06.15.1) [15] for protein identification and spectral counts were computed by Prohits (version 6.0.4) [16]. Spectral counts were transferred in Perseus (Version 1.6.1.3) [17]. Proteins quantified in three out of four experiments for either WT or R103H POLR3B were kept for further analysis. Spectral counts reported as 0 by X-Tandem were replaced by a randomly generated spectral count value normally distributed with a mean and S.D. equal to those of the lowest $20 \%$ spectral count values from the LC-MS/MS analysis. Spectral counts were normalized by the spectral count of the bait (POLR3B) in order to allow comparison between different purifications. WT and R103H POLR3B proteins were compared to the FLAG empty vector control samples and were labeled as high-confidence interactors when their $p$ value was under 0.05 and their spectral count ratio was over 2. Statistical differences between WT and R103H POLR3B were determined using a two-tailed T-test subsequently adjusted for multiple hypothesis testing using a permutation-based test by considering a False discovery rate (FDR) of 5\% adjusted using an s0 correction factor of 0.1 with 10,000 iterations [18]. The level of differential interaction with POLR3B were considered statistically significant when the adjusted $P$ value was $<0.05$ and its average spectral count fold-change (R103H/WT) was \pm 2 .

\section{Behavioral tests}

Six months old WT and DM mice were submitted to the balance beam, rotarod and open field tests as previously described [6]. Male (5 WT, 3 DM) and female (3 WT, 5 DM) mice were used for the balance beam and rotarod tests, while an independent cohort of males (7 WT, $8 \mathrm{DM}$ ) were subsequently used for the open field test.

\section{Histology}

For preparation of tissue sections, mouse cerebrum and cerebellum were harvested and fixed in $4 \%$ paraformaldehyde for $24 \mathrm{~h}$ at $4{ }^{\circ} \mathrm{C}$. Tissues were then equilibrated in $30 \%$ sucrose/PBS for $48 \mathrm{~h}$. Sagittal or coronal sections $(30 \mu \mathrm{m})$ were cut using a freezing sledge microtome. Free-floating sections were processed for immunofluorescence as previously described [19]. Sections were labeled with an antibody against PLP (Abcam \#ab28486, $1 / 200$ ) in three mice per group. Nissl stains and Purkinje cell counts were performed in two mice per group as previously described [20]. Imaging was performed using a Zeiss Axiovert M2 microscope.

\section{RNA extraction, RT-PCR and Northern blots}

Cerebral and cerebellar hemispheres were harvested and snap-frozen in liquid nitrogen. Tissues were homogenized in Qiazol lysis reagent (Qiagen). Total RNA was extracted with the miRNeasy kit (Qiagen) and treated with DNAse I (Qiagen) according to the manufacturer's instructions. For RT-PCR of Polr3b exons 4-8, brain RNA was reversed transcribed into complementary DNA (cDNA) using the Superscript III Reverse Transcriptase (ThermoFisher) according to the manufacturer's instructions and amplified by PCR using the following primers: 5'-CAGCACATAGACTCCTTT AACTATTTC-3'and 5'-GGGTGGAGCTGGTAACTGA3 '. Northern Blots for precursor and mature tRNAs were performed as previously described [6].

\section{RNA-sequencing}

Illumina TruSeq rRNA-depleted stranded libraries were prepared from total cerebrum RNA of 6 months old male mice ( $3 \mathrm{WT}, 3 \mathrm{DM}$ ) and sequenced on an Illumina HiSeq 2500 with $125 \mathrm{bp}$ paired-end reads at an average of 38.7 million reads/sample. Quality control and trimming were performed as previously described [21]. Trimmed reads were aligned to the reference genome mm10 using STAR v2.3.0e [22]. Expression levels were estimated with featureCounts [23] using exonic reads and normalized using DESeq2 [24]. Differential expression analysis was performed with DESeq2. 


\section{Additional files}

Additional file 1: Figure S1. FLAG-POLR3B R103H and FLAG-POLR3B WT expression levels. (PDF $2856 \mathrm{~kb}$ )

Additional file 2: Table S1. AP-MS data for POLR3B WT and R103H (XLSX $22 \mathrm{~kb})$

\section{Abbreviations}

AP-MS: Affinity purification coupled to mass spectrometry; cDNA: Complementary DNA; CNS: Central nervous system; DM: Double mutant mice; HLD: Hypomyelinating leukodystrophy; MRI: Magnetic resonance imaging; ncRNA: Non-coding RNA; PLP: Proteolipid protein; Pol III: RNA Polymerase III; rRNA: Ribosomal RNA; SEM: Standard error of the mean; tRNA: Transfer RNA; WT: Wild-type

\section{Acknowledgements}

We would like to thank Eve-Marie Charbonneau and Geneviève Hamel from the Douglas Neurophenotyping Platform for their help with mouse colony management and behavioral assays and Dr. Joseph Rochford for assistance with analysis of behavioral data. We also wish to acknowledge the McGill University and Génome Québec Innovation Center for sequencing services and the IRCM Mass Spectrometry and Proteomics core facility for proteomics services. BC holds a Bell-Bombardier chair awarded by the IRCM.

\section{Authors' contributions}

$\mathrm{KC}$ and $\mathrm{BB}$ conceived the study. KC, RDM, RL, MSG, BC, IMW, CLK and BB designed the experiments. KC, SY, RDM, MJD, NS and RL performed experiments and analyzed data. KC and CLK analyzed RNA-seq data. MP generated AP-MS data. MP, CP, MSG and BC analyzed AP-MS data. KC and BB wrote the manuscript. All authors read and approved the final manuscript.

\section{Funding}

This project was supported by grants from the Fondation Leuco Dystrophie, the European Leukodystrophy Association and the Canadian Institutes of Health Research (CIHR) (MOP \#126141). The mouse line C57BL/6 N-Polr3b< em4Tcp > was made as part of the NorCOMM2 project, funded by the Care4Rare Canada Consortium, Genome Canada and the Ontario Genomics Institute (OGI-051) at the Toronto Centre for Phenogenomics. KC received doctoral awards from the Fonds de Recherche en Santé - Québec (FRQS), the Fondation Grand Défi Pierre Lavoie and the Jewish General Hospital National Bank Molecular Pathology Scholarship. CLK receives a salary award from the FRQS.

\section{Availability of data and materials}

The datasets used and/or analysed during the current study are available from the corresponding author on reasonable request.

\section{Ethics approval}

All experiments were performed according to good practice of handling laboratory animals consistent with the Canadian Council on Animal Care and approved by the McGill University Animal Care Committee.

\section{Consent for publication}

Not applicable.

\section{Competing interests}

The authors declare that they have no competing interests.

\section{Author details}

${ }^{1}$ Montreal Neurological Institute, McGill University, 3801 University Street, room 622, Montréal, Québec H3A 2B4, Canada. ²Department of Human Genetics, McGill University, Québec, Montréal, Canada. ${ }^{3}$ Lady Davis Institute for Medical Research, Jewish General Hospital, Québec, Montréal, Canada. ${ }^{4}$ Translational Proteomics Laboratory, Institut de recherches cliniques de Montréal (IRCM), Québec, Montréal, Canada. ${ }^{5}$ Department of Biochemistry, Albert Einstein College of Medicine, New York, Bronx, USA. ${ }^{6}$ Département de biochimie et médecine moléculaire, Université de Montréal, Québec, Montréal, Canada.
Received: 25 March 2019 Accepted: 11 June 2019

Published online: 20 June 2019

\section{References}

1. Bernard G, Chouery E, Putorti ML, Tetreault M, Takanohashi A, Carosso G, Clement I, Boespflug-Tanguy O, Rodriguez D, Delague V, et al. Mutations of POLR3A encoding a catalytic subunit of RNA polymerase pol III cause a recessive hypomyelinating leukodystrophy. Am J Hum Genet. 2011;89:415-23.

2. Tetreault M, Choquet $K$, Orcesi S, Tonduti D, Balottin U, Teichmann M, Fribourg S, Schiffmann R, Brais B, Vanderver A, Bernard G. Recessive mutations in POLR3B, encoding the second largest subunit of pol III, cause a rare hypomyelinating leukodystrophy. Am J Hum Genet. 2011;89:652-5.

3. Thiffault I, Wolf NI, Forget D, Guerrero K, Tran LT, Choquet K, Lavallee-Adam M, Poitras C, Brais B, Yoon G, et al. Recessive mutations in POLR1C cause a leukodystrophy by impairing biogenesis of RNA polymerase III. Nat Commun. 2015;6:7623.

4. Dorboz I, Dumay-Odelot H, Boussaid K, Bouyacoub Y, Barreau P, Samaan S, Jmel H, Eymard-Pierre E, Cances C, Bar C, et al. Mutation in POLR3K causes hypomyelinating leukodystrophy and abnormal ribosomal RNA regulation. Neurol Genet. 2018;4:e289.

5. Wolf NI, Vanderver A, van Spaendonk RM, Schiffmann R, Brais B, Bugiani M, Sistermans E, Catsman-Berrevoets C, Kros JM, Pinto PS, et al. Clinical spectrum of $4 \mathrm{H}$ leukodystrophy caused by POLR3A and POLR3B mutations. Neurology. 2014;83:1898-905.

6. Choquet K, Yang S, Moir RD, Forget D, Lariviere R, Bouchard A, Poitras C, Sgarioto N, Dicaire MJ, Noohi F, et al. Absence of neurological abnormalities in mice homozygous for the Polr3a G672E hypomyelinating leukodystrophy mutation. Mol Brain. 2017:10:13.

7. Sievers F, Wilm A, Dineen D, Gibson TJ, Karplus K, Li W, Lopez R, McWilliam $H$, Remmert M, Soding J, et al. Fast, scalable generation of high-quality protein multiple sequence alignments using Clustal omega. Mol Syst Biol. 2011;7:539.

8. Choquet K, Forget D, Meloche E, Dicaire MJ, Bernard G, Vanderver A, Schiffmann R, Fabian MR, Teichmann M, Coulombe B, et al. Leukodystrophyassociated POLR3A mutations down-regulate the RNA polymerase III transcript and important regulatory RNA BC200. J Biol Chem. 2019;294: 7445-59.

9. Vanderver A, Tonduti D, Bernard G, Lai J, Rossi C, Carosso G, Quezado M, Wong K, Schiffmann R. More than hypomyelination in pol-III disorder. J Neuropathol Exp Neurol. 2013;72:67-75.

10. Tiedge $\mathrm{H}$, Chen W, Brosius J. Primary structure, neural-specific expression, and dendritic location of human BC200 RNA. J Neurosci. 1993;13:2382-90.

11. Shin H, Lee J, Kim Y, Jang S, Lee Y, Kim S, Lee Y. Knockdown of BC200 RNA expression reduces cell migration and invasion by destabilizing mRNA for calcium-binding protein S100A11. RNA Biol. 2017;14:1418-30.

12. Singh R, Gupta SC, Peng WX, Zhou N, Pochampally R, Atfi A, Watabe K, Lu $Z$, Mo YY. Regulation of alternative splicing of Bcl-x by BC200 contributes to breast cancer pathogenesis. Cell Death Dis. 2016:7:e2262.

13. Skryabin BV, Sukonina V, Jordan U, Lewejohann L, Sachser N, Muslimov I, Tiedge $\mathrm{H}$, Brosius J. Neuronal untranslated BC1 RNA: targeted gene elimination in mice. Mol Cell Biol. 2003;23:6435-41.

14. Chiu YH, Macmillan JB, Chen ZJ. RNA polymerase III detects cytosolic DNA and induces type I interferons through the RIG-I pathway. Cell. 2009;138:576-91.

15. Craig R, Beavis RC. TANDEM: matching proteins with tandem mass spectra. Bioinformatics. 2004:20:1466-7.

16. Liu G, Zhang J, Larsen B, Stark C, Breitkreutz A, Lin ZY, Breitkreutz BJ, Ding Y, Colwill K, Pasculescu A, et al. ProHits: integrated software for mass spectrometry-based interaction proteomics. Nat Biotechnol. 2010;28:1015-7.

17. Tyanova S, Temu T, Sinitcyn P, Carlson A, Hein MY, Geiger T, Mann M, Cox J. The Perseus computational platform for comprehensive analysis of (prote)omics data. Nat Methods. 2016;13:731-40.

18. Tusher VG, Tibshirani R, Chu G. Significance analysis of microarrays applied to the ionizing radiation response. Proc Natl Acad Sci U S A. 2001:98:5116-21.

19. Lariviere R, Gaudet R, Gentil BJ, Girard M, Conte TC, Minotti S, LeclercDesaulniers K, Gehring K, McKinney RA, Shoubridge EA, et al. Sacs knockout mice present pathophysiological defects underlying autosomal recessive spastic ataxia of Charlevoix-Saguenay. Hum Mol Genet. 2015;24:727-39.

20. Girard M, Lariviere R, Parfitt DA, Deane EC, Gaudet R, Nossova N, Blondeau F, Prenosil G, Vermeulen EG, Duchen MR, et al. Mitochondrial dysfunction and Purkinje cell loss in autosomal recessive spastic ataxia of CharlevoixSaguenay (ARSACS). Proc Natl Acad Sci U S A. 2012;109:1661-6. 
21. Antonicka H, Choquet K, Lin ZY, Gingras AC, Kleinman CL, Shoubridge EA. A pseudouridine synthase module is essential for mitochondrial protein synthesis and cell viability. EMBO Rep. 2017;18:28-38.

22. Dobin A, Davis CA, Schlesinger F, Drenkow J, Zaleski C, Jha S, Batut $P$, Chaisson M, Gingeras TR. STAR: ultrafast universal RNA-seq aligner. Bioinformatics. 2013;29:15-21.

23. Liao Y, Smyth GK, Shi W. featureCounts: an efficient general purpose program for assigning sequence reads to genomic features. Bioinformatics. 2014;30:923-30

24. Love Ml, Huber W, Anders S. Moderated estimation of fold change and dispersion for RNA-seq data with DESeq2. Genome Biol. 2014;15:550.

\section{Publisher's Note}

Springer Nature remains neutral with regard to jurisdictional claims in published maps and institutional affiliations.

Ready to submit your research? Choose BMC and benefit from:

- fast, convenient online submission

- thorough peer review by experienced researchers in your field

- rapid publication on acceptance

- support for research data, including large and complex data types

- gold Open Access which fosters wider collaboration and increased citations

- maximum visibility for your research: over $100 \mathrm{M}$ website views per year

At BMC, research is always in progress.

Learn more biomedcentral.com/submissions 